\title{
Vorwort
}

Soncino - eine Stadt in der oberitalienischen Provinz Cremona wurde zum Namensgeber einer aus Deutschland eingewanderten jüdischen Druckerfamilie, die sich hohe Verdienste im hebräischen Buchdruck im 15. und 16. Jahrhundert erwarb. Kaum bekannt ist, dass sich nach jener Druckerfamilie die erste und einzige jüdische Bibliophilen-Vereinigung benannte: die Soncino-Gesellschaft der Freunde des jüdischen Buches e.V., die sich 1924 in Berlin gründete. Während ihres 13-jährigen Bestehens betrug ihre Mitgliederzahl über 800 und war damit im Vergleich zu anderen bibliophilen Vereinigungen in der Weimarer Republik ausgesprochen hoch. Zu ihren Mitgliedern zählten zahlreiche prominente und einflussreiche Persönlichkeiten aus allen Bereichen des gesellschaftlichen Lebens. Umso überraschender ist, dass die Soncino-Gesellschaft heute nur wenigen bekannt ist, mit Ausnahme bibliophiler Sammler und des internationalen Antiquariatshandels, wo die Publikationen der Gesellschaft nach wie vor einen klangvollen Namen haben. Während die Bücher das Interesse der Sammler wecken, ist die Geschichte der Gesellschaft nur wenig erforscht.

Als Erster skizzierte Abraham Horodisch ${ }^{1}$, einer der Gründer der SoncinoGesellschaft, 1963 allein auf sein Gedächtnis gestützt seine Erinnerungen an die Gründung und Tätigkeit der Vereinigung in der Festschrift für Carl Wehmer ${ }^{2}$, die später in überarbeiteter Form erneut publiziert wurden. ${ }^{3}$ Bis heute ist sein Aufsatz die primäre Quelle, auf die sich alle späteren Publikationen beziehen.

Im selben Jahr veröffentlichte Fritz Homeyer ${ }^{4}$ den schmalen Band Deutsche Juden als Bibliophilen und Antiquare (1963), der auch den Wiederabdruck des ersten veröffentlichten Mitgliederverzeichnisses der Soncino-Gesellschaft enthält.

Der spiritus rector der Gesellschaft, Herrmann Meyer $^{5}$, erinnerte kurz vor seinem Tod 1972 ebenfalls in einem kurzen Beitrag im Börsenblatt für den Deutschen Buchhandel an seine Zeit als Schriftführer und Redakteur der Soncino-

1 Horodisch, Abraham: Ein Abenteuer im Geiste. Die Soncino-Gesellschaft der Freunde des jüdischen Buches. In: Jost, Siegfried (Hrsg.): Bibliotheca Docet. Festgabe für Carl Wehmer. Amsterdam 1963, S. 181-208.

2 Carl Wehmer (1903-1978), Frühdruckforscher und Direktor der Universitätsbibliothek Heidelberg.

3 Horodisch, Abraham: Die Soncino-Gesellschaft der Freunde des jüdischen Buches. In: Imprimatur, Neue Folge V (1967), S. 131-148.

4 Homeyer, Fritz: Deutsche Juden als Bibliophilen und Antiquare. Tübingen 1963. Drei Jahre später erschien die Schrift in einer zweiten, erweiterten und verbesserten Auflage.

5 Meyer, Herrmann: Als Antiquar in Jerusalem. In: Börsenblatt für den Deutschen Buchhandel - Frankfurter Ausgabe 52 (30. Juni 1972), S. A 218-A 220. 


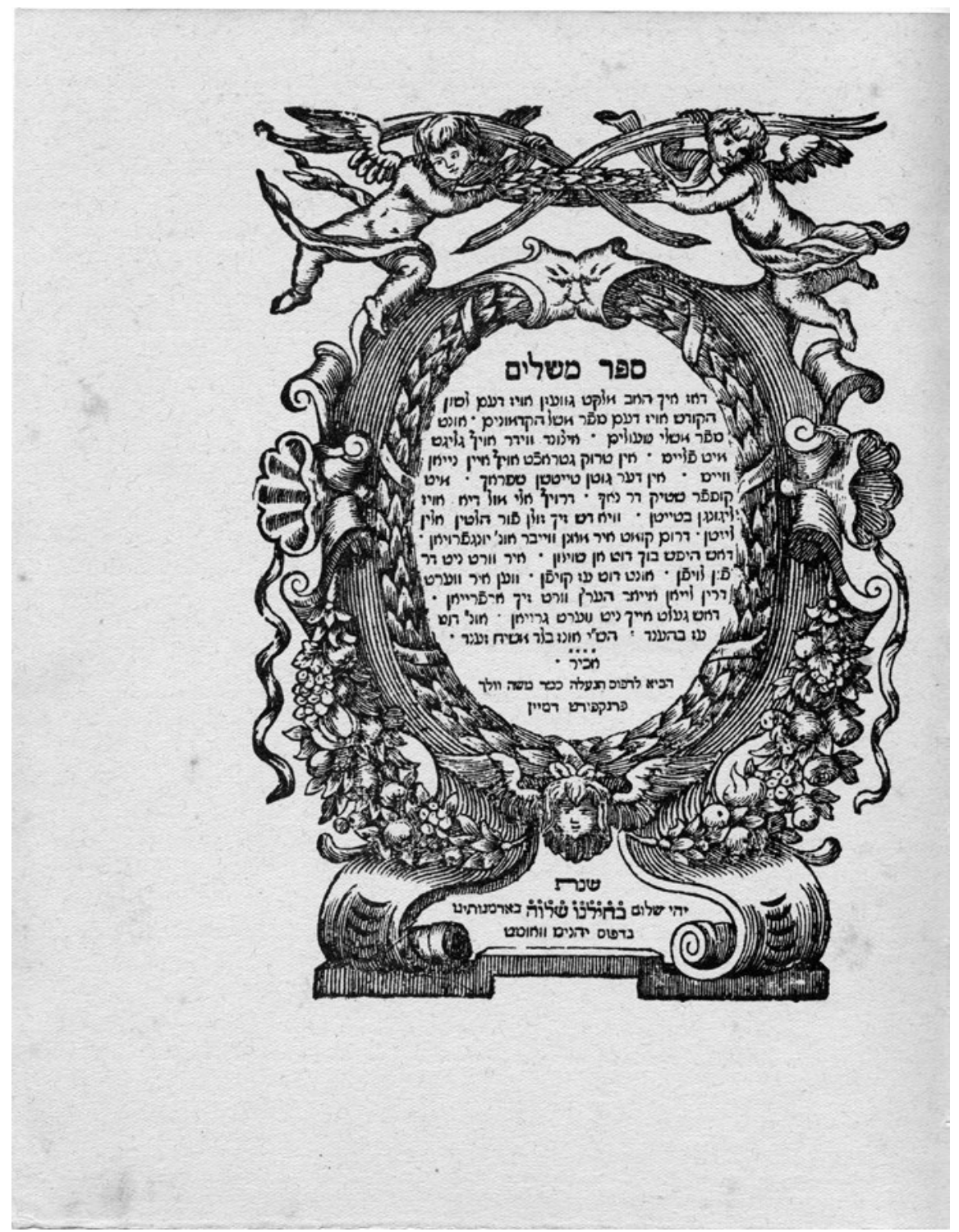

Abb. 1: Titelblatt des Sefer Meschalim genannt das Kuhbuch, Faksimile-Reproduktion zur ersten Publikation der Soncino-Gesellschaft, 1925. 
Blätter. Meyer bewahrte sicher nicht nur alle seinen Wirkungskreis betreffenden Schriftstücke auf, sondern sammelte auch sämtliche von der Gesellschaft hergestellten Drucke, ein Vereinsarchiv im eigentlichen Sinne führte er jedoch nicht. Unterlagen, Schriftstücke und Korrespondenzen darüber hinaus, die als Vereinsarchiv gelten könnten, konnten bislang nicht aufgefunden werden.

Meyers Nachlass wurde für das Jüdische Museum Berlin während dessen Aufbauphase erworben und befindet sich seitdem im Archiv des Jüdischen Museums Berlin. Es ist die bislang umfangreichste und vermutlich einzige ergiebige Quelle für Forschungen zur Soncino-Gesellschaft.

Einen ersten lexikalischen Eintrag zur Soncino-Gesellschaft gab es bereits 1930 im vierbändigen Jüdischen Lexikon, offensichtlich befördert von den Begründern des Lexikons, Georg Herlitz und Bruno Kirschner, die wie Josef Meisl und Felix Theilhaber als Mitarbeiter der Redaktion, Soncino-Mitglieder waren. ${ }^{6}$ Auch der bis in die letzte Auflage der Encyclopaedia Judaica 2007 existente Eintrag der Gesellschaft wurde ebenfalls von einem Gründer der Gesellschaft, Herrmann Meyer, verfasst, ergänzt um aktuelle bibliographische Angaben. ${ }^{7}$

1931 erstellte Julius Rodenberg im Rahmen der Veröffentlichungen der Deutschen bibliophilen Gesellschaften die erste Bibliographie der Soncino-Gesellschaft mit den bis dahin erschienenen Publikationen. ${ }^{8}$

Quellen zu Informationen vereinsinterner Debatten, dem Fortgang von Druckvorhaben, Erinnerungen an Personen und Ereignisse finden sich vereinzelt in veröffentlichten Briefwechseln, sind jedoch vermutlich in den weltweit verstreuten Nachlässen ehemaliger Protagonisten zu finden. Hier eröffnen sich Felder für weitere Forschungsarbeiten.

In den letzten drei Jahrzehnten hat das Interesse an der Forschung zur jüdischen Buchkultur vor dem Zivilisationsbruch des 20. Jahrhunderts zugenommen. 1987 veröffentlichte Lothar Sommer als Beitrag für die Pirckheimer Gesellschaft einen Überblick über Berliner bibliophile Vereine in der Zeit von der Jahrhundertwende bis 1945. ${ }^{9}$ Basierend auf den bereits erwähnten Quellen erscheint darin ein län-

6 Vgl. Jüdisches Lexikon. Ein enzyklopädisches Handbuch des jüdischen Wissens in vier Bänden. Band IV/2 S-Z. Berlin 1930, S. $494 \mathrm{f}$.

7 Vgl. Encyclopaedia Judaica. Second Edition. Volume 19, SOM-TN, Jerusalem 2007, S. 11.

8 Rodenberg, Julius: Die Soncino-Gesellschaft der Freundes des jüdischen Buches e.V./Berlin. In: Deutsche Bibliophilie in drei Jahrzehnten. Verzeichnis der Veröffentlichungen der deutschen bibliophilen Gesellschaft und der ihr gewidmeten Gaben 1898-1930, hrsg. von der Deutschen Bücherei. Leipzig 1931, S. 199-210.

9 Sommer, Lothar: Berliner bibliophile Vereine in der Zeit von der Jahrhundertwende bis 1945. Bedeutung und Grenzen. Ein Überblick. In: Marginalien. Zeitschrift für Buchkunst und Bibliophilie 106 (1987), hrsg. von der Pirckheimer Gesellschaft, S. 1-53 [insbesondere S. 27-35]. 
gerer Abschnitt über die Soncino-Gesellschaft, der erstmals die Entwicklung des Vereins der Freunde des jüdischen Buches beschreibt und innerhalb der anderen bibliophilen Vereinigungen verortet.

Der Buchwissenschaftler Ernst Fischer ${ }^{10}$ eröffnete in seinem Beitrag „Zerstörung einer Buchkultur“ die Debatte neu, indem er auf Abraham Horodisch und Herrmann Meyer als bislang einzige Quellen verweist und damit das Forschungsdesiderat deutlich macht. Biographische Details einiger führender Soncino-Mitglieder und Quellenverweise finden sich auch in seinem biographischen Handbuch Verleger, Buchhändler und Antiquare aus Deutschland und Österreich in der Emigration nach 1933. ${ }^{11}$

In seinem Überblickswerk Jüdische Kultur in der Weimarer Republik (2000) ${ }^{12}$ geht Michael Brenner aus kulturhistorischer Sicht umfassender auf die SoncinoGesellschaft ein und verweist darauf, dass die jüdischen Bibliophilen im Gegensatz zu den Reformjuden „kein Interesse an einer Neudefinition des traditionellen Kanons heiliger jüdischer Bücher oder Gebete“13 hatten.

Markus Kirchhoff hat mit seiner Publikation Häuser des Buches (2002) der jüdischen Buchkultur ein Denkmal gesetzt, in der auch die Soncino-Gesellschaft eine angemessene Darstellung findet. ${ }^{14}$

Dem Antiquar Ulrich Heider in Köln ist das Verdienst zuzuschreiben, aus Anlass der 2. Kölner Antiquariatstage 2006 ein Bändchen in bibliophiler Form herausgegeben zu haben, das fußend auf den biographischen Quellen Horodischs und Meyers den Versuch unternimmt, das bisherige Wissen zu bündeln und neu zu bewerten. ${ }^{15}$ Trotz einiger Fehler ist Heiders Publikation ein wichtiger Impuls für die wissenschaftliche Beschäftigung mit dem Thema geworden. Daran ändert auch die kritische Analyse des Heider-Bandes von Rainer Fürst und Klaus Schreiber nichts. ${ }^{16}$

10 Fischer, Ernst: Zerstörung einer Buchkultur. Die Emigration jüdischer Büchersammler aus Deutschland nach 1933 und ihre Folgen. In: Imprimatur, Neue Folge XVII (Juni 2002), S. 176-195. 11 Ders.: Verleger, Buchhändler und Antiquare aus Deutschland und Österreich in der Emigration nach 1933. Ein biographisches Handbuch, hrsg. vom Verband Deutscher Antiquare. Elbingen 2011.

12 Die amerikanische Originalausgabe, vgl. Brenner, Michael: The Renaissance of Jewish Culture in Weimar Germany. New Haven and London 1996.

13 Ders.: Jüdische Kultur in der Weimarer Republik. München 2000, S. 193.

14 Kirchhoff, Markus: Häuser des Buches. Bilder jüdischer Bibliotheken. Leipzig 2002, S. 105108.

15 Heider, Ulrich: Die Soncino-Gesellschaft der Freunde des jüdischen Buches e. V. (1924-1937). Köln 2006.

16 Fürst, Rainer und Klaus Schreiber: Die Soncino-Gesellschaft der Freunde des jüdischen Buches e. V. (1924-1937). In: Informationsmittel 2 (2007): http://swbplus.bsz-bw.de/bsz266595642rez.htm (14.10.2013). 
AuchamSalomon-Ludwig-Steinheim-Institut für deutsch-jüdische Geschichte wird seit Jahren das Thema hebräischer Buchdruck im kulturgeschichtlichen Kontext beforscht. Im Rahmen dessen ist 2007 von Harald Lordick der Beitrag „Schlag mich in van Geldern ein!“ erschienen. ${ }^{17}$

Nicht unerwähnt bleiben darf die hervorragende Publikation Das jüdische Buch im Dritten Reich (1993) von Volker Dahm, die zwar nicht explizit auf die Soncino-Gesellschaft eingeht, aber aufzeigt, dass die Entwicklung der jüdischen Buchkultur nicht abrupt 1933 endete: „Mit der Macht im Staate fiel den Nationalsozialisten nicht automatisch die Herrschaft über das kulturelle Leben zu. " ${ }^{18}$ Dies gilt auch für die Soncino-Gesellschaft.

\section{Zu diesem Band}

Die vorliegende Publikation ist aus Anlass der Gründung der Soncino-Gesellschaft vor 90 Jahren entstanden. Sie stellt in acht Essays die Geschichte der Soncino-Gesellschaft der Freunde des jüdischen Buches sowie einige wichtige Protagonisten vor. Es werden ihr Engagement für die hebräische Buchkultur beschrieben, das Netzwerk ihrer Mitglieder beleuchtet sowie ein zionistischer Einfluss und die Wirkungsgeschichte der Vereinigung hinterfragt. Die Intention der Herausgeberinnen war es, aus kulturhistorischer Perspektive diese bis heute singuläre Gründung einer jüdischen Bibliophilen-Gesellschaft zu betrachten und weitere Forschungen anzuregen.

In ihrem einführenden Essay geben Karin Bürger, Ines Sonder und Ursula Wallmeier einen Überblick über die Entwicklung der Gesellschaft, wobei ein besonderes Augenmerk auf die Zeit nach 1933 gelegt wird, die in den bisherigen Darstellungen bislang kaum Beachtung fand und in der wechselnd von Auflösung, Zerschlagung oder von einer Weiterführung der Arbeit unter reglementierenden Bedingungen die Rede ist.

Frank Schlöffel widmet sich in seinem Beitrag „Zionismus und Bibliophilie“ einem der wichtigsten Protagonisten der Soncino-Gesellschaft, dem Publizisten und Bibliothekar Heinrich Loewe. Er gehörte zum ersten gewählten Vorstand und fungierte von 1926 bis zu seiner Übersiedlung nach Palästina 1933 als Vorsitzender der Vereinigung, deren „Seele“ er laut Abraham Horodisch war. Loewe, der schon 1905 die Gründung einer Jüdischen Nationalbibliothek in Jerusalem vorge-

17 Lordick, Harald: Schlag mich in van Geldern ein! Freude am schönen Buch und die SoncinoGesellschaft. In: Kalonymos. Beiträge zur deutsch-jüdischen Geschichte aus dem Salomon Ludwig Steinheim-Institut 2-3 (2007), S. 1-4.

18 Dahm, Volker: Das jüdische Buch im Dritten Reich. 2., überarb. Aufl. München 1993, S. 26. 
schlagen hatte, war auch einer der Hauptakteure im Hinblick auf eine hebräische Kulturarbeit innerhalb der zionistischen Bewegung, in deren Kreisen er um Interessenten als Mitglieder für die neuen „Soncinaten“ warb, zu denen später eine Reihe bekannter zionistischer Persönlichkeiten und Körperschaften gehörten.

Vor dem Hintergrund des typografischen Diskurses innerhalb der deutschen Buchkunst- und Pressenbewegung zur Zeit der Weimarer Republik untersucht der Beitrag „Von der Form der Sprache“ von Philipp Messner die von der Soncino-Gesellschaft ausgehende Initiative für eine neue hebräische Buchschrift als besonderes Phänomen der hebräischen Renaissance und Kulturarbeit. Im Zentrum der Untersuchung stehen dabei die Debatten um den Entwurf einer neuen hebräischen Drucktype für die Herausgabe der Soncino-Bibel, mit deren typographischer Gestaltung der nichtjüdische Schriftkünstler Marcus Behmer beauftragt worden war.

Martin Münzel analysiert in seinem Beitrag „Zwischen Ökonomie und Bibliophilie“ am Beispiel der Unternehmer und Verleger eine spezifische Gruppe innerhalb der Soncino-Gesellschaft, die als Repräsentanten der Wirtschaft und Mitglieder einer Bibliophilen-Gesellschaft bislang nicht im Zentrum der (kultur-) historischen Forschung standen. In einem separaten Diskurs wird am Beispiel Kurt Enochs eine Verlegerpersönlichkeit vorgestellt, der nach seiner Emigration in die Vereinigten Staaten trotz marktorientierter Verlagsstrategien im Taschenbuchformat mit qualitätsbewussten Ansprüchen als der „bibliophile Mr. Paperback“ bekannt und erfolgreich wurde.

Zwei Essays beschäftigen sich mit Publikationen der Soncino-Gesellschaft, ihrer Entstehungsgeschichte und ihren wichtigsten Akteuren.

Ines Sonder stellt in ihrem Beitrag „Neun Holzschnitte zum Buch Jesus Sirach“ die neunte Publikation der Soncino-Gesellschaft von 1929 vor: Jakob Steinhardt Neun Holzschnitte zu ausgewählten Versen aus dem Buche Jeschu ben Elieser ben Sirah. Dabei wird der Bogen von der Auffindung der Fragmente zum Buch Jesus Sirah 1890 in der Genisah der Synagoge in Alt-Kairo bis zu den Illustrationen des Druckgraphikers Jakob Steinhardt gespannt. Die Publikation war aufgrund ihrer besonderen typographischen Gestaltung eine „druckerische Herausforderung“ und kann laut Abraham Horodisch als „der erste moderne bibliophile hebräische Druck“ gelten, der „mit den Maßstäben westeuropäischer Buchkunst gemessen werden kann“. 19

Jutta Dick geht in ihrem Beitrag „Frucht vom Baum des Lebens“ auf die letzte ordentliche Veröffentlichung der Soncino-Gesellschaft Frucht vom Baum des Lebens. Ozer Peroth Ez Chajim. Die Sammlung der Rechtsgutachten Peri Ez Chajim des Rabbinerseminars Ets Haim zu Amsterdam (1937) ein, die 1937 von Menko

19 Horodisch, Soncino-Gesellschaft (wie Anm. 3), S. 136. 
Max Hirsch herausgegeben wurde. Die Rechtsgutachten waren zwischen 1691 und 1807 von dem portugiesischen Rabbinerseminar Ets Haim in Amsterdam in 13 Bänden gedruckt worden. Menko Max Hirsch, der aus der bekannten Halberstädter Familie Hirsch stammte, hatte 1928 mit der Edition und Übersetzung der Rechtsgutachten begonnen, die neun Jahre später im Exil in Amsterdam erschienen.

Ihren Essay „Deutsch-jüdische Buchkultur zwischen Sammlung und Zerstreuung. Von Berlin nach Tel Aviv“ widmet Verena Lenzen dem Berliner Bibliophilen Gotthard Laske und seinem Sohn Ernst Laske. Der Konfektionsfabrikant Gotthard Laske gehörte seit 1926 zu den neuen Vorstandsmitgliedern der Soncino-Gesellschaft, die 1931 auch den Druck der Soncino-Bibel unterzeichneten. Im November 1936 nahm er sich in Berlin das Leben, seine 10.000 Bände umfassende Bibliothek wurde über die ganze Welt verstreut, Teile davon konnten nach Palästina gerettet werden. Dorthin war sein Sohn Ernst Laske nach mehreren Emigrationsstationen Anfang 1948 eingewandert. Ende der 1970er Jahre übernahm er in Tel Aviv die deutschsprachige Buchhandlung Landsberger's Bookshop (gegründet 1930). Nach seinem Tod wurden die geretteten Bücher aus der Sammlung seines Vaters verauktioniert.

Regina Thiele stellt in ihrem Beitrag „... ich zog nun werbend durch Berlin“ den Nachlass Herrmann Meyer im Archiv des Jüdischen Museums Berlin vor. Aus archivwissenschaftlicher Sicht beschreibt sie die Bestandsbildung und den Inhalt der Sammlung Herrmann Meyer, ergänzt um biographische Informationen zum Gründer und Schriftführer der Soncino-Gesellschaft. Der Nachlass ist bis heute eine der wenigen umfassenden Quellen zur Vereinsgeschichte.

Der Anhang des Bandes ergänzt die Beiträge durch eine umfassende Liste der Soncino-Mitglieder versehen mit einer ausführlichen Einleitung, eine Bibliographie der Publikationen der Soncino-Gesellschaft ist erweitert um ein Register der Personen und Druckereien sowie eine Auflistung der von Herrmann Meyer gesammelten Presseausschnitte zur Gründung und Entwicklung der Gesellschaft.

Die Herausgeberinnen danken den Mitarbeitern des Archivs und der Bibliothek des Jüdischen Museums Berlin, dem Salomon-Ludwig-Steinheim-Institut für deutsch-jüdische Geschichte an der Universität Duisburg-Essen und dem Archiv der Akademie der Künste, Berlin, Ulf Preuß vom Digitalisierungslabor der Fachhochschule Potsdam sowie allen weiteren Institutionen, die bei Recherchen hilfreich waren.

Unser ausdrücklicher Dank gilt Frau Josefa Bar Or-Steinhardt, Naharija (Israel), Herrn Martin Rosenthal, Berlin, sowie Julius H. Schoeps für seine Anregung zu diesem Band. Des Weiteren gilt unser Dank Sarah Jaglitz, Heike Hilbert, Amelie Bäcker und Natalie Römer. Unserem Autor Frank Schlöffel danken wir 
zudem für seine Bereitschaft, uns weiterführendes Quellenmaterial zur Verfügung gestellt zu haben.

Potsdam, im März 2014

Karin Bürger, Ines Sonder, Ursula Wallmeier

Die Herausgeberinnen haben sich entschieden, Personenbezeichnungen aus Gründen der besseren Lesbarkeit weitgehend in der männlichen Form zu verwenden, weibliche Personen sind darin ausdrücklich eingeschlossen. 